\title{
The diagnosis of coccidioidomycosis
}

\author{
Neil M Ampel
}

Address: Southern Arizona Veterans Affairs Health Care System, Tucson, AZ 85723, USA

Email: nampel@email.arizona.edu

FI000 Medicine Reports 2010, 2:2 (doi:10.3410/M2-2)

The electronic version of this article is the complete one and can be found at: http://fl000.com/reports/medicine/content/2/2

\begin{abstract}
Until recently, culture, microscopy, and serology have been the available methods for the diagnosis of coccidioidomycosis. While Coccidioides is frequently isolated by culture, special precautions must be taken because of the risk of laboratory infection and because Coccidioides is on the Select Agent list. Serology is useful but the sensitivity remains lower than desired. A commercially available test for coccidioidal galactomannan antigenuria now exists and appears useful for immunocompromised hosts with severe disease. Polymerase chain reaction assays targeting specific coccidioidal genes have demonstrated utility but are not commercially available. Moreover, their sensitivity and the best sample type remain unestablished.
\end{abstract}

\section{Introduction and context}

The endemic region for coccidioidomycosis is restricted to the Western Hemisphere. Almost all coccidioidal infections are due to inhalation of arthroconidia, 2-5 $\mu \mathrm{m}$ barrel-shaped spores that dislodge from hyphae and become airborne [1]. A full $60 \%$ of all coccidioidal infections are completely asymptomatic, with only the expression of specific delayed-type hypersensitivity as a marker for infection [2]. Unfortunately, coccidioidal skin tests are not currently available in the US.

The other $40 \%$ of individuals with acute pulmonary coccidioidomycosis present with a syndrome similar to a bacterial community-acquired pneumonia, with cough, pleuritic chest pain, and fever. In one recent study performed in the coccidioidal endemic region, coccidioidomycosis was misidentified in approximately $29 \%$ of individuals as a bacterial process [3]. In fewer than $1 \%$ of all individuals infected, disseminated clinical infection occurs outside of the thoracic cavity. This most commonly presents as a skin or soft tissue lesion, osteomyelitis, or meningitis.

Coccidioidomycosis is now recognized to be caused by two distinct species, Coccidioides immitis and C. posadasii. The former appears to be geographically limited to the southern portion of California and Northern Mexico while the latter occurs in all geographic areas [4]. At this time, the presentation of disease and diagnosis do not appear to differ between the two species.

\section{Available diagnostic tests}

For the past several decades three modalities - culture, microscopy, and serology - have been the mainstays of specific diagnosis of coccidioidomycosis.

\section{Culture}

Unlike other endemic fungi, Coccidioides grows readily on a variety of culture media at $35^{\circ} \mathrm{C}$ and is usually visually apparent in 2-7 days. It can be presumptively identified based on its early lack of pigmentation and the later presence of septate hyphae with barrel-shaped arthroconidia on microscopic examination [5]. A proprietary nucleic acid chemiluminescent probe (AccuProbe, Gen-Probe, Inc., San Diego, CA, USA) allows rapid, specific identification once visible growth has occurred [6]. A major drawback of culture is the extreme danger of Coccidioides in the laboratory. Several documented accidental exposures have occurred and Stevens and colleagues [7] have recently outlined their approach to managing such events. Moreover, Coccidioides is listed as a 'Select Agent' of bioterrorism [8]. Because of these 
issues, suspected and established cultures of Coccidioides should be handled using Biosafety Level 3 containment.

\section{Microscopy}

Coccidioides can be identified directly in fresh respiratory secretions using potassium hydroxide, calcifluor, and Papanicolaou. However, the sensitivity of these methods is relatively low $[9,10]$. The diagnosis can also be established in fixed tissue using a variety of stains, including hematoxylin-eosin and Gomori methenamine-silver. The pathognomonic structure is the spherule.

\section{Serology}

Serologic tests for the diagnosis of coccidioidomycosis are time honored and perhaps the most frequently employed assays for the diagnosis of coccidioidomycosis $[1,11]$. Smith and colleagues established the modern use of coccidioidal serology with the development of the tube precipitin (TP) and complement fixation (CF) assays [12]. Each relies on a specific antigen. For TP, this is now known to be a heat-stable $120 \mathrm{kDa} \beta$ glucosidase [13], while for CF it is a heat-labile chitinase [14]. The IDTP and IDCF are variants of these assays that employ immunodiffusion (ID) in agar. All are very specific and, at least from early studies, relatively sensitive [12]. The TP and IDTP assays conform to an immunoglobulin (Ig)M reaction and are positive early in illness and, at times, during recurrence. The CF and IDCF assays become positive usually after 2-3 weeks of illness, conform to an IgG reaction, and their titer has prognostic implications, with high titers portending severe or disseminated disease. In persons who resolve their clinical illness, both reactions become negative over time.

\section{Recent advances Serology}

Since the 1990s, a proprietary enzyme-linked immunosorbant assay (Premier EIA, Meridian Diagnostics, Inc., Cincinnati, OH, USA) has been widely employed in the endemic region. Martins and colleagues [15] initially compared this assay to standard TP and CF assays as well as the less specific latex agglutination assay and, after combining the IgM and IgG enzyme immunoassay (EIA) results, found that the specificity was $98.5 \%$ and the sensitivity was 95.5\%. Kaufman and coworkers [16] examined 47 specimens from persons with confirmed coccidioidomycosis and a positive IDCF and found all were also positive for either IgM or IgG using the EIA; however, 12 of 345 samples from patients with other pulmonary infections were also EIA positive. In another study, among 41 patients with culture-confirmed coccidioidomycosis who had three serologic tests performed, the CF was positive in 23 (56\%), the IDCF in 29 (71\%), and the EIA in 34 (81\%) [17]. In six (15\%) patients, all serologic tests were negative. Blair and colleagues [18] examined immunocompromised patients and found, as expected, that serologic tests were less likely to be positive than among normal hosts. They subsequently examined patients with an isolated IgM EIA reaction and were able to confirm a diagnosis of coccidioidomycosis by other means in all cases [20]. Hence, while there is lingering concern regarding the specificity of the Premier EIA, particularly the IgM (D Pappagianis, personal communication), published reports suggest that it is sensitive. Another proprietary EIA (Coccidioides DxSelect ${ }^{\mathrm{TM}}$, Focus Diagnostics, Inc., Cypress, CA, USA) became available in 2006. There are no published studies on its performance parameters.

\section{Detection of antigenuria}

During the 1980s, assays were developed to detect coccidioidal circulating antigen [20-22]. Unfortunately, these never achieved the sensitivity required for clinical use. However, Kuberski and coworkers [23] recently reported several instances where a commercially available assay for detecting Histoplasma capsulatum antigenuria was found to be positive in samples from patients with coccidioidomycosis. This led to the development of a specific assay for Coccidioides [24]. To develop this test, antibody was raised in rabbits immunized to galactomannan isolated from the mycelial stage of clinical isolates of Coccidioides and used in an enzyme-linked immunosorbent assay. The assay detected as little as $0.03 \mathrm{ng} / \mathrm{ml}$ of Coccidioides galactomannan. Among 24 patients with coccidioidomycosis, most with underlying immunosuppression and severe disease, 17 (71\%) had a positive test. The Histoplasma antigenuria assay was positive in 14 of these patients while coccidioidal antigenuria was detected in 3 of 28 (11\%) samples from patients with other endemic mycoses, including two with histoplasmosis, confirming the problem of cross-reactivity.

\section{Genomic analysis}

Tests based on detection of the genetic fingerprint of the fungus offer the promise of rapidity and specificity without the requirement of growing the organism or relying on the response of the host. While several investigators have demonstrated the feasibility of such approaches, no such tests are currently commercially available.

Because of high copy number and variability among fungal species, one region of particular interest in such analyses has been the $18 \mathrm{~S}$ ribosomal rRNA genomic sequence (rDNA). Johnson and coworkers [25] used primers for this region in a polymerase chain reaction (PCR) to determine if the coccidioidal rDNA genome 
could be detected in human sera obtained from patients with suspected coccidioidomycosis. Among 94 samples tested, all originally submitted for coccidioidal serologic testing, 6 demonstrated an appropriately sized 239 basepair DNA band. Using these same primers, Japanese investigators were recently able to detect this band in DNA extracted from formalin-fixed paraffin-embedded lung tissue from four patients with coccidioidomycosis [26].

Binnicker and colleagues [27] employed real-time PCR with primers amplifying a 170 base-pair region in the ITS2 (internal transcribed spacer 2) region of the rDNA genome to examine 266 respiratory specimens submitted for fungal culture. Most of these specimens were submitted from within the coccidioidal endemic region. Among 20 samples that were culture positive for Coccidioides, 16 were positive by real-time PCR, compared to none of the culture negative samples, yielding a sensitivity of $98 \%$ and a specificity of $100 \%$. In addition, real-time PCR was positive in all 47 samples found to demonstrate Coccidioides by histopathological examination, whereas it was positive in 17 cases among 101 where histopathology was negative.

Bialek and colleagues [28] have performed several studies using PCR to detect the unique coccidioidal gene, antigen-2/proline rich antigen (Ag2/PRA) [29,30], which exists as only one copy in the genome. They initially correctly identified 120 isolates of C. posadasii from Mexico using both conventional nested and realtime PCR and found no false positives [28]. Since then, Brazilian investigators were able to apply nested PCR to a respiratory sample from a patient with pulmonary coccidioidomycosis and detected the appropriate 349 base-pair product of Ag2/PRA in the sample [31].

\section{Implications for clinical practice}

The concerns regarding the specificity of Premier EIA compared to the older serologic assays are likely to persist. For the present, a TP or IDTP should be obtained for all positive IgM EIA tests, and a CF or IDCF assay should be obtained and titrated in all instances of a positive EIA IgG. However, experts recommend that the EIA is very sensitive and other assays need not be obtained if it is negative [32]. It is also clear that a serologic test may be negative during active coccidioidomycosis and serology should not be wholly relied upon in such instances.

A critical question regarding coccidioidal antigenuria is its sensitivity for different syndromes of coccidioidomycosis. While preliminary data suggest it is helpful for those who are highly immunocompromised and for those with widely disseminated disease, it is not clear if it will be useful for those with less severe illness and for those with disease confined to the thoracic cavity. In addition, there is documented cross-reactivity, particularly in patients with histoplasmosis, which may reduce its diagnostic utility.

Finally, while genomic detection of Coccidioides is promising, much more work on sensitivity and sample type needs to be done. Moreover, PCR has been notoriously difficult to perform in clinical laboratories because of the risk of contamination. These issues will need to be resolved prior to these assays becoming clinically available.

\section{Abbreviations}

Ag2/PRA, antigen-2/proline rich antigen; $\mathrm{CF}$, complement fixation; EIA, enzyme immunoassay; ID, immunodiffusion; Ig, immunoglobulin; ITS2, internal transcribed spacer 2; PCR, polymerase chain reaction; rDNA, ribosomal DNA; TP, tube precipitin.

\section{Competing interests}

The author declares that he has no competing interests.

\section{References}

I. Pappagianis D, Zimmer BL: Serology of coccidioidomycosis. Clin Microbiol Rev 1990, 3:247-68.

2. Smith CE, Beard RR, Whiting EG, Rosenberger HG: Varieties of coccidioidal infection in relation to the epidemiology and control of the diseases. Am J Public Health 1946, 36:1394-402.

3. Valdivia L, Nix D, Wright M, Lindberg E, Fagan T, Lieberman D, Stoffer T, Ampel NM, Galgiani JN: Coccidioidomycosis as a common cause of community-acquired pneumonia. Emerg Infect Dis 2006, I2:958-62.

4. Fisher MC, Koenig GL, White TJ, Taylor JW: Molecular and phenotypic description of Coccidioides posadasii sp. nov., previously recognized as the non-California population of Coccidioides immitis. Mycologia 2002, 94:73-84.

5. Sutton DA: Diagnosis of coccidioidomycosis by culture: safety considerations, traditional methods, and susceptibility testing. Ann N Y Acad Sci 2007, I I I I:3 I5-25.

6. Padhye AA, Smith G, Standard PG, McLaughlin D, Kaufman L: Comparative evaluation of chemiluminescent DNA probe assays and exoantigen tests for rapid identification of Blastomyces dermatitidis and Coccidioides immitis. J Clin Microbiol 1994, 32:867-70.

7. Stevens DA, Clemons KV, Levine HB, Pappagianis D, Baron EJ, Hamilton JR, Deresinski SC, Johnson N: Expert opinion: what to do when there Is Coccidioides exposure in a laboratory. Clin Infect Dis 2009, 49:919-23.

8. Dixon DM: Coccidioides immitis as a select agent of bioterrorism. J Appl Microbiol 2001, 91:602-5.

9. DiTomasso JP, Ampel NM, Sobonya RE, Bloom JW: Bronchoscopic diagnosis of pulmonary coccidioidomycosis. Comparison of cytology, culture, and transbronchial biopsy. Diagn Microbiol Infect Dis 1994, 18:83-7.

10. Sarosi GA, Lawrence JP, Smith DK, Thomas A, Hobohm DW, Kelley PC: Rapid diagnostic evaluation of bronchial washings in patients with suspected coccidioidomycosis. Semin Respir Infect 2001, 16:238-41

II. Pappagianis D: Serologic studies in coccidioidomycosis. Semin Respir Infect 2001, 16:242-50. 
12. Smith CE, Saito MT, Simons SA: Pattern of 39,500 serologic tests in coccidioidomycosis. J Am Med Assoc 1956, I60:546-52.

13. Hung CY, Yu JJ, Lehmann PF, Cole GT: Cloning and expression of the gene which encodes a tube precipitin antigen and wallassociated beta-glucosidase of Coccidioides immitis. Infect Immun 2001, 69:2211-22.

14. Johnson SM, Pappagianis D: The coccidioidal complement fixation and immunodiffusion-complement fixation antigen is a chitinase. Infect Immun 1992, 60:2588-92.

15. Martins TB, Jaskowski TD, Mouritsen CL, Hill HR: Comparison of commercially available enzyme immunoassay with traditional serological tests for detection of antibodies to Coccidioides immitis. J Clin Microbiol 1995, 33:940-3.

16. Kaufman L, Sekhon AS, Moledina N, Jalbert M, Pappagianis D: Comparative evaluation of commercial Premier EIA and microimmunodiffusion and complement fixation tests for Coccidioides immitis antibodies. J Clin Microbiol 1995, 33:618-9.

17. Polage CR, Billetdeaux E, Litwin CM, Petti CA: Revisiting the sensitivity of serologic testing in culture positive coccidioidomycosis [Abstract F-005]. Presented at the $106^{\text {th }}$ General Meeting of the American Society for Microbiology: May 2006: Orlando.

18. Blair JE, Coakley B, Santelli AC, Hentz JG, Wengenack NL: Serologic testing for symptomatic coccidioidomycosis in immunocompetent and immunosuppressed hosts. Mycopathologia 2006, 162:317-24.

19. Blair JE, Currier JT: Significance of isolated positive IgM serologic results by enzyme immunoassay for coccidioidomycosis. Mycopathologia 2008, 166:77-82.

20. Galgiani JN, Dugger KO, Ito JI, Wieden MA: Antigenemia in primary coccidioidomycosis. Am J Trop Med Hyg 1984, 33:645-9.

21. Wack EE, Dugger KO, Galgiani JN: Enzyme-linked immunosorbent assay for antigens of Coccidioides immitis: human sera interference corrected by acidification-heat extraction. J Lab Clin Med 1988, I I I:560-5.

22. Weiner $\mathrm{MH}$ : Antigenemia detected in human coccidioidomycosis. J Clin Microbiol 1983, 18:136-42.
23. Kuberski T, Myers R, Wheat LJ, Durkin M, Connolly P, Kubak BM, Bruckner D, Pegues D: Diagnosis of coccidioidomycosis by antigen detection using cross-reaction with a Histoplasma antigen. Clin Infect Dis 2007, 44:e50-4.

24. Durkin M, Connolly P, Kuberski T, Myers R, Kubak BM, Bruckner D, Pegues D, Wheat LJ: Diagnosis of coccidioidomycosis with use of the Coccidioides antigen enzyme immunoassay. Clin Infect Dis 2008, 47:e69-73.

FI000 Factor 3.0 Recommended

Evaluated by Cornelia Lass-Floerl 19 Nov 2008

25. Johnson SM, Simmons KA, Pappagianis D: Amplification of coccidioidal DNA in clinical specimens by PCR. J Clin Microbiol 2004, 42: 1982-5.

26. Kishi K, Fujii T, Takaya H, Miyamoto A, Kurosaki A, Kohno T, Yoshimura K: Pulmonary coccidioidomycosis found in healthy Japanese individuals. Respirology 2008, 13:252-6.

27. Binnicker MJ, Buckwalter SP, Eisberner JJ, Stewart RA, McCullough AE, Wohlfiel SL, Wengenack NL: Detection of Coccidioides species in clinical specimens by real-time PCR. J Clin Microbiol 2007, 45:173-8.

28. Bialek R, Kern J, Herrmann T, Tijerina R, Ceceñas L, Reischl U, González GM: PCR assays for identification of Coccidioides posadasii based on the nucleotide sequence of the antigen $2 /$ proline-rich antigen. J Clin Microbiol 2004, 42:778-83.

29. Dugger KO, Villareal KM, Ngyuen A, Zimmermann CR, Law JH, Galgiani JN: Cloning and sequence analysis of the cDNA for a protein from Coccidioides immitis with immunogenic potential. Biochem Biophys Res Commun 1996, 21 8:485-9.

30. Zhu Y, Yang C, Magee DM, Cox RA: Coccidioides immitis antigen 2: analysis of gene and protein. Gene 1996, I8I:12I-5.

31. de Aguiar Cordeiro R, Nogueira Brilhante RS, Gadelha Rocha MF, Araujo Moura FE, Pires de Camargo Z, Costa Sidrim J]: Rapid diagnosis of coccidioidomycosis by nested PCR assay of sputum. Clin Microbiol Infect 2007, I3:449-5I.

32. Saubolle MA, McKellar PP, Sussland D: Epidemiologic, clinical, and diagnostic aspects of coccidioidomycosis. J Clin Microbiol 2007. 45:26-30. 